\title{
DETECTION OF LONGITUDINAL WAVES IN RESONANCE WITH CAPILLARY WAVES AT THE AIR-WATER INTERFACE BY ENERGY TRANSFER
}

WOLFGANG BUDACH AND DIETMAR MÖBIUS

Max-Planck-Institut für Biophysikalische Chemie, Am Fassberg, 3400 Göttingen (F.R.G.)

(Received April 25, 1989; accepted May 25, 1989)

Capillary (transverse) ripples are generated at a monolayer-covered air-water interface. Compression of the monolayer changes the surface dilatational modulus $\varepsilon$ and the reduced elasticity $\varepsilon / \sigma$ of the monolayer. When the reduced elasticity reaches a particular value, resonance between the capillary wave and the longitudinal wave should occur. Longitudinal waves (lateral oscillations, modulation of the average distances of the molecules) are detected by using energy transfer (Förster type) between dye molecules incorporated in the monolayer. Capillary ripples are detected by specular reflection of a laser beam from the water surface. The dependence of the longitudinal and the transverse wave characteristics on the area per molecule was investigated, and the resonance phenomenon was observed near the theoretical value of $\varepsilon / \sigma$ for the resonance condition.

\section{INTRODUCTION}

Capillary waves have been investigated at the monolayer-covered air-water interface by electrostatic deflection ${ }^{1}$. Under particular conditions this tranverse mode is in resonance with the longitudinal mode ${ }^{2,3}$, which can be considered as a periodic change in the surface density of the amphiphilic molecules. Characterization of both wave types is very sensitive to interference of the detector with the air-water interface. Thus the propagation of both wave modes has been studied using optical methods which do not disturb the surface mechanically and allow the determination of wavelength and damping constant. The propagation of the capillary wave has been determined by the analysis of the reflection of a laser beam under normal incidence ${ }^{1,4,5}$. In this paper we report the propagation characteristics of longitudinal waves in a mixed monolayer containing an energy transfer couple, which we monitored by measuring the sensitized fluorescence of the energy acceptor at $500 \mathrm{~nm}$ after excitation of the energy donor at $366 \mathrm{~nm}$ and distance-dependent Förster-type lateral energy transfer ${ }^{6}$. By scanning the reflected laser beam and the sensitized acceptor fluorescence in the propagation direction, both wavelength and damping constant of the two wave modes have been determined at different surface pressures, or areas per molecule. The results are compared with the hydrodynamic theory. 


\section{THEORY}

The hydrodynamic theory predicts as a result of the resonance between transverse and longitudinal modes of surface excitation a maximum of the damping constant of the capillary wave while the amplitude of the longitudinal wave has a maximum ${ }^{7}$. Lucassen has shown that resonance occurs when the surface dilatational modulus satisfies the condition $\varepsilon=\varepsilon_{\mathrm{m}}$ with the following expression for the surface dilatational modulus ${ }^{2,7,8}$ :

$$
\varepsilon_{\mathrm{m}}=\left(2 \omega^{3} \rho \eta_{\mathrm{s}}\right)^{1 / 2} / k^{2}
$$

Calculation of the reduced elasticity $\varepsilon / \sigma$ from eqn.(1) yields the value 0.09 (dimensionless; with $v=60 \mathrm{~Hz}, \eta_{\mathrm{s}}=10 \mathrm{mP}, \lambda=5 \mathrm{~mm}, \rho=1000 \mathrm{~kg} \mathrm{~m}^{-3}$ and $\sigma=72 \mathrm{mN} \mathrm{m}^{-1}$ ).

Combination of the dispersion relations of capillary and longitudinal waves ${ }^{2,4}$ under the resonance condition ( $k=k_{\mathrm{L}}=k_{\mathrm{T}}$, same generation frequency $v$ ) leads to the following equation for the reduced elasticity at resonance:

$$
\frac{\varepsilon}{\sigma}=\left(\frac{1}{2}+2^{-3 / 2}\right)\left(\frac{\eta_{\mathrm{s}}{ }^{2} k}{\rho \sigma}\right)^{1 / 4}
$$

This equation yields the value 0.06 with the parameters given above. We have also calculated the reduced elasticity of the monolayer from $\pi-A$ isotherm data.

Longitudinal waves modulate the average distance between the energy donor and the energy acceptor molecule incorporated in the monolayer. The efficiency of the energy transfer process depends on the distance between donor and acceptor molecules ${ }^{9}$, and thus the presence of a longitudinal wave should lead to the modulation of the sensitized fluorescence of the acceptor molecule. The coupling of the longitudinal mode may be qualitatively represented by the extrapolated amplitude $\xi_{0}$ of the fluorescence intensity modulation at the site of electrostatic generation. Energy transfer ${ }^{10,11}$ has been investigated in biological membranes including the theoretical treatment of lateral transfer in two-dimensional systems $^{12,13}$.

\section{EXPERIMENTAL SECTION}

\subsection{Excitation and detection of capillary waves}

The transverse waves are generated by the electrostatic deflection of the interface with an inhomogeneous electric field between a metal blade and the water surface (blade: $80 \mathrm{~mm}$ long, $0.02 \mathrm{~mm}$ thick; distance between blade and water surface, less than $1 \mathrm{~mm})$. For the excitation a sinusoidal voltage $(60 \mathrm{~Hz}, 125 \mathrm{~V})$ is applied to the blade ${ }^{1}$. The $\mathrm{He}-\mathrm{Ne}$ laser beam is focused on the water surface and the reflection angle is measured with a differential photodiode. The wave characteristics are determined by scanning along the propagation direction (stroboscopic method) ${ }^{14}$.

\subsection{Excitation and detection of longitudinal waves}

The longitudinal waves are generated without contacting the interface by resonance with the capillary mode. The fluorescence intensity measurements are 
made by using a specially designed quartz fibre optics head with an extension of $0.3 \mathrm{~mm}$ in the propagation direction of the waves (less than $1 / 10$ of the wavelength) and $80 \mathrm{~mm}$ perpendicular to it. The donor molecule $S 9$ (the structure is shown in Fig. 1) is excited with a mercury lamp at $366 \mathrm{~nm}$. The modulation of the acceptor fluorescence (S8; for structure see Fig. 1) by the longitudinal wave is detected at $500 \mathrm{~nm}$ using the stroboscopic method. Interference filters are used to select both excitation and detection wavelengths.
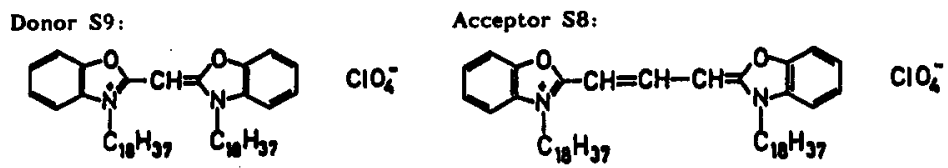

Fig. 1. Structures of the acceptor and donor molecules $N^{\prime}, N$-dioctadecyloxacyanine perchlorate (donor, S9) and $N^{\prime}, N$-dioctadecyloxacarbocyanine perchlorate (acceptor, S8).

\subsection{Monolayer preparation}

The subphase used was Milli-Q water buffered at $\mathrm{pH} 10.6$ with $0.01 \mathrm{M}$ phosphate buffer. The optical and spectroscopic system was adjusted and the wave characteristics on the clean water surface were determined. Then a mixed chloroform solution $\left(5 \mathrm{mM}\right.$ ) with $\mathrm{S} 8: \mathrm{S9}: \mathrm{C}_{20}=1: 1: 50$ (matrix molecule: $\mathrm{C}_{20}$, arachidic acid) was spread. Subsequently the monolayer was compressed stepwise to given surface pressures (measured by a Wilhelmy balance) and the wave characteristics were determined. The complete system was mounted on a vibrationally damped table.

\section{DETECTION OF CAPILLARY AND LONGITUDINAL WAVES}

Figure 2 shows the propagation characteristics of a capillary wave in the presence of the mixed monolayer $S 8: S 8: C_{20}=1: 1: 50$. Figure 3 shows the measured

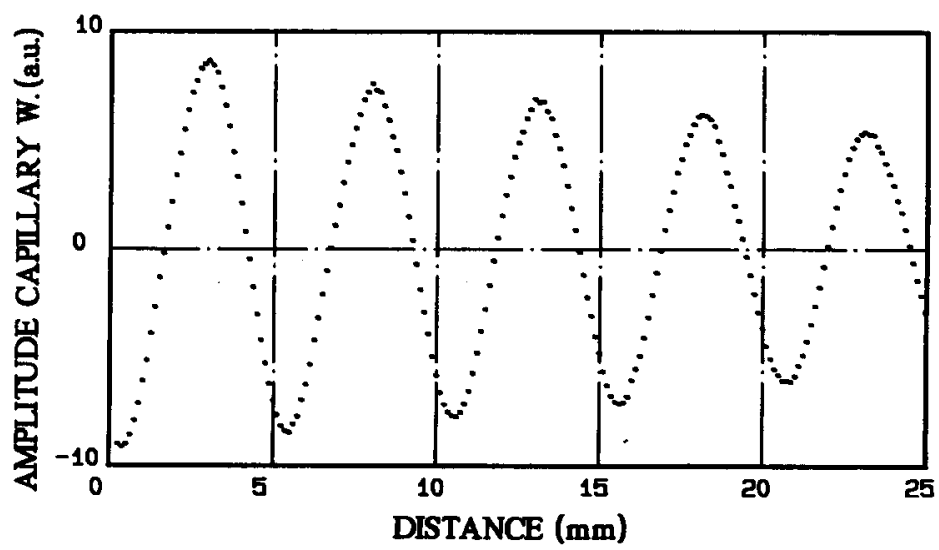

Fig. 2. Propagation characteristics of a capillary wave at a monolayer-covered air-water interface. The amplitude $\zeta$ (arbitrary units) of the transverse wave is plotted $v$ s. scanning distance $(0 \mathrm{~mm}$ represents the arbitrary start position of the detection system) parallel to the propagation direction. Monolayer S8:S9: $\mathbf{C}_{20}=1: 1: 50 ;$ surface pressure, $1 \mathrm{mN} \mathrm{m}^{-1}$. 


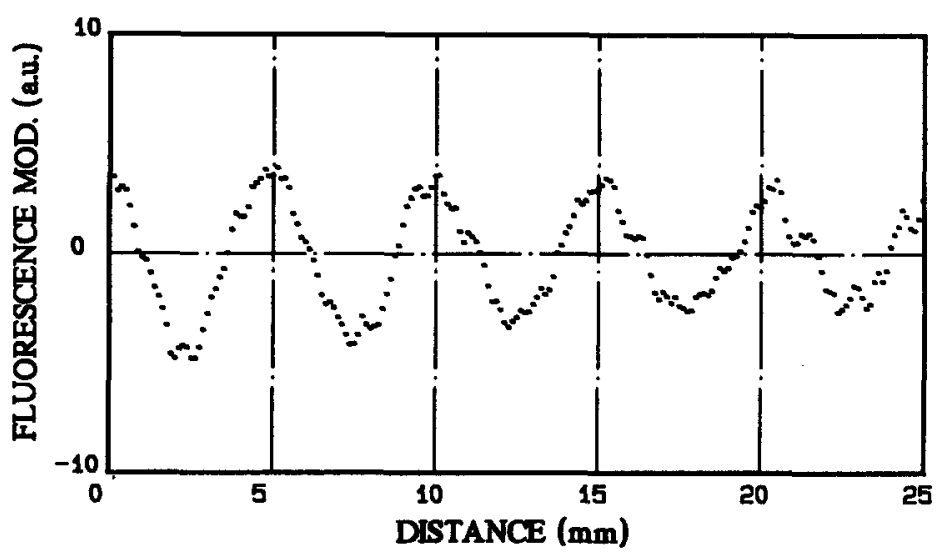

Fig. 3. Modulation of the sensitized acceptor fluorescence $\xi$ (arbitrary units) due to lateral periodic oscillations of the molecules parallel to the propagation direction plotted $v$ s. scanning distance $(0 \mathrm{~mm}$ represents the arbitrary start position of the detection system). Monolayer $\mathrm{S} 8: \mathrm{S} 9: \mathrm{C}_{20}=1: 1: 50$ surface pressure, $1 \mathrm{mN} \mathrm{m}^{-1}$.

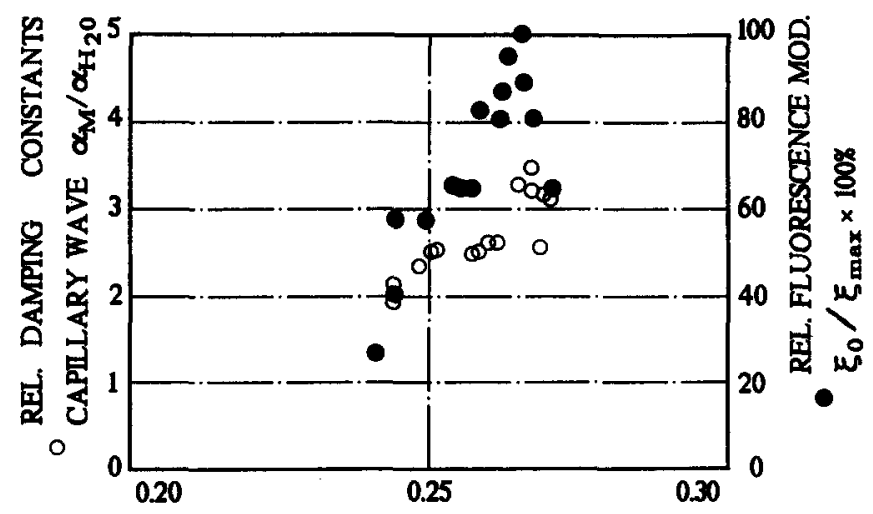

AREA / MATRIX MOLECULE (nm 2$)$

Fig. 4. $O$, relative damping constants $\alpha_{\mathrm{M}} / \alpha_{\mathrm{H}_{2} \mathrm{O}}$ plotted $v s$. area per matrix molecule; $\bullet$, extrapolated fluorescence intensity amplitude $\xi_{0}$ (see Fig. 3) at zero scanning distance related to the strongest observed modulation $\xi_{\max }$.

modulation of the sensitized acceptor fluorescence. For the capillary waves, the relative damping constants $\alpha_{\mathrm{M}} / \alpha_{\mathrm{H}_{2} \mathrm{O}}$ are plotted $v$ s. area per matrix molecule in Fig. 4 (circles). The full circles in Fig. 4 show the fluorescence intensity modulation amplitude extrapolated to scanning distance of $0 \mathrm{~mm}\left(\xi_{0}\right.$, shown in Fig. 3) related to the strongest modulation $\xi_{\max }$. The strongest damping of the capillary waves is observed between 0.26 and $0.27 \mathrm{~nm}^{2}$ for the area per matrix molecule where the sensitized acceptor fluorescence modulation also has a maximum. The occurrence of maxima in both plots for areas per matrix molecule between 0.26 and $0.27 \mathrm{~nm}^{2}$ is due to the resonance between the two wave modes. In this range the calculated reduced elasticity of the monolayer is about $\varepsilon / \sigma=0.1$. According to eqns. (1) and (2) resonance should occur when the reduced elasticity is between 0.06 and 0.09 . 


\section{CONCLUSIONS}

Longitudinal waves have been generated in monolayers at the air-water interface via resonance to capillary (transverse) waves. Lateral energy transfer between donor and acceptor molecules incorporated in the monolayer is modulated by the passage of the longitudinal waves. This enables the determination of the characteristics of longitudinal wave propagation. The resonance phenomenon was observed at a low surface dilatational modulus. This is in agreement with the theoretical predictions.

\section{REFERENCES}

C. H. Sohl, K. Miyano and J. B. Ketterson, Rev. Sci. Instrum., 49 (1978) 1464.

J. Lucassen, Trans. Faraday Soc., 64 (1968) 2221.

3 R. Cini, P. P. Lombardini, C. Manfredi and E. Cini, J. Colloid Interface Sci., 119 (1987) 1.

4 V. Vogel and D. Möbius, Langmuir, 5 (1989) 129.

5 R. S. Hansen and J. Ahmad, in J. F. Danielli, M. D. Rosenberg and D. A. Cadenhead (eds.), Progress in Surface Membrane Science, Vol. 4, Academic Press, New York, 1971, pp. 1-56.

6 H. Kuhn, D. Möbius and H. Bücher, in A. Weissberger and B. Rossiter (eds.), Physical Methods of Chemistry, Vol. 1, Part IIIB, Wiley, New York, 1972, p. 577.

7 E. H. Lucassen-Reynders and J. Lucassen, Adv. Colloid Interface Sci., 92 (1983) 297.

8 D. Thiesen and A. Sheludko, Kolloid-Z., 218 (1967) 139.

9 Y. Yonezawa, D. Möbius and H. Kuhn, J. Appl. Phys., 62 (1987) 2022.

10 Th. Förster, Ann. Phys., 2 (1947) 55.

11 Th. Förster, Fluoreszenz Organischer Verbindungen, Vandenhoeck and Ruprecht, Göttingen, 1st edn., 1950, pp. 182, 321.

12 T. G. Dewey and G. G. Hammes, Biophys. J., 32 (1980) 1023.

13 B. Synder and E. Freire, Biophys. J., 40 (1982) 137.

14 R. S. Hansen and J. A. Mann, Jr., J. Appl. Phys., 35 (1964) 158.

15 R. C. Stone and W. J. Rice, J. Colloid Interface Sci., 61 (1) (1977) 160.

\section{APPENDIX A: NOMENCLATURE}

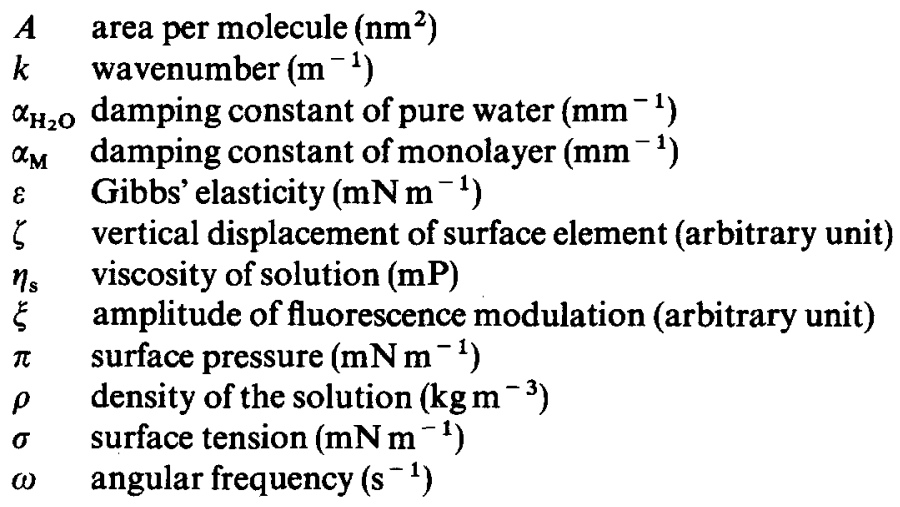

